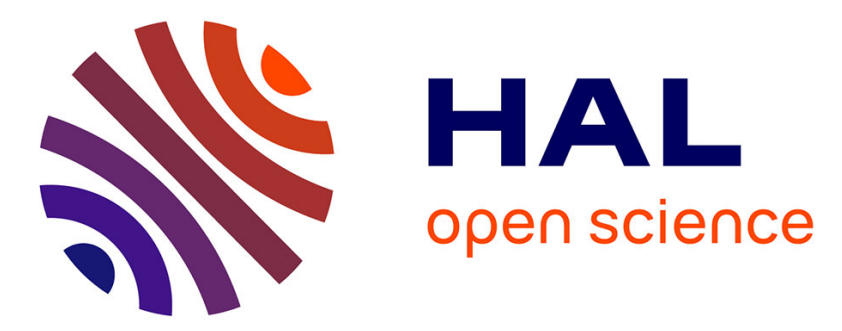

\title{
Aza-boron-dipyrromethene dyes: TD-DFT benchmarks, spectral analysis and design of original near-IR structures
}

\author{
Boris Le Guennic, Olivier Maury, Denis Jacquemin
}

\section{To cite this version:}

Boris Le Guennic, Olivier Maury, Denis Jacquemin. Aza-boron-dipyrromethene dyes: TD-DFT benchmarks, spectral analysis and design of original near-IR structures. Physical Chemistry Chemical Physics, 2011, 14 (1), pp.157-164. 10.1039/C1CP22396H . hal-00753767

\section{HAL Id: hal-00753767 https://hal.science/hal-00753767}

Submitted on 6 Sep 2013

HAL is a multi-disciplinary open access archive for the deposit and dissemination of scientific research documents, whether they are published or not. The documents may come from teaching and research institutions in France or abroad, or from public or private research centers.
L'archive ouverte pluridisciplinaire HAL, est destinée au dépôt et à la diffusion de documents scientifiques de niveau recherche, publiés ou non, émanant des établissements d'enseignement et de recherche français ou étrangers, des laboratoires publics ou privés. 


\title{
Aza-boron-dipyrromethene dyes: TD-DFT benchmarks, spectral analysis and design of original near-IR structures $\dagger$
}

\author{
Boris Le Guennic, ${ }^{* a b}$ Olivier Maury ${ }^{a}$ and Denis Jacquemin $* c$ \\ Received 24th July 2011, Accepted 17th October 2011 \\ DOI: $10.1039 / \mathrm{c1cp22396h}$
}

The excited-state energies of aza-boron-dipyrromethene (Aza-BODIPY) derivatives are investigated with Time-Dependent Density Functional Theory (TD-DFT), with twin goals. On the one hand, a pragmatic, yet efficient, computational protocol is defined in order to reach rapidly semi-quantitative estimates of the $\lambda_{\max }$ of these challenging dyes. It turned out that a PCM-TD-BMK/6-311+G(2d,p)//PCM-PBE0/6-311G(2d,p) approach delivers appropriate lower bounds of the experimental results, despite the inherent limits of the vertical approximation. On the other hand, the method is applied to design new dyes absorbing in the near-IR. The spectral features of $c a .30$ new compounds have been simulated in a systematic way, trying to efficiently combine several available synthetic strategies leading to significant bathochromic displacements. A series of dyes absorbing above $850 \mathrm{~nm}$ are proposed, illustrating that (relatively) fast theoretical calculations might be a useful pre-screening step preceding synthesis.

\section{Introduction}

Whereas primer applications of near-infrared (NIR) dyes mainly concerned optical recording, laser printing and filters, ${ }^{1,2}$ such chromophores found recently new applications in various fields such as bioimaging, sensing, photodynamic therapy, photovoltaics, organic light emitting diodes, advanced optoelectronics, and nonlinear optics. ${ }^{3-6}$ At a prerequisite to the emergence of such technologies, chemists had to concentrate their effort on the design and synthesis of new NIR chromophores with optimised spectroscopic properties in the 700-1200 nm spectral range. Among the whole panel of applications of NIR luminescent dyes, particular attention was focused on in vivo microscopy imaging ${ }^{4}$ since the NIR region is less absorbed and scattered by biological tissues, allowing deeper penetration and higher contrast. Unfortunately, the emission red-shift up to the NIR is usually accompanied by a strong decrease of the luminescence quantum yield $(\Phi)$. As a consequence, the main challenge consists

\footnotetext{
${ }^{a}$ Université de Lyon, CNRS, Institut de Chimie de Lyon, Ecole Normale Supérieure de Lyon, 15 Parvis René Descartes, 69342 Lyon Cedex 07, France

${ }^{b}$ Laboratoire des Sciences Chimiques de Rennes,

UMR 6226 CNRS-Université de Rennes 1, 35042 Rennes Cedex,

France.E-mail: boris.leguennic@univ-rennes1.fr

${ }^{c}$ Chimie Et Interdisciplinarité, Synthèse, Analyse, Modélisation

(CEISAM), UMR CNRS 6230, BP 92208, Université de Nantes, 2 , Rue de la Houssinière, 44322 Nantes Cedex 3, France.

E-mail:denis.jacquemin@univ-nantes.fr

$\dagger$ Electronic supplementary information (ESI) available: Tables with the results of basis set investigation for spectra and geometry. List of structural parameters and wavelengths computed with all functionals. Position of all major bands of $\mathbf{4}$ and 5. TD benchmarks for 1-3. See DOI: $10.1039 / \mathrm{clcp} 22396 \mathrm{~h}$
}

in the synthesis of chromophores featuring high quantum yield beyond $700 \mathrm{~nm}$. Despite their low photostability in the biological environment and, for most of them, modest quantum yields, cyanine dyes are still the most frequently used chromophores. ${ }^{1,2}$ Recently, boron-dipyrromethene (referred as BODIPY in the following) dyes were considered as a possible alternative strategy, since this subclass of cyanine dyes features a sharp fluorescence emission band with a large quantum yield and a higher photoand chemostability. ${ }^{7,8}$ Unfortunately, absorption and emission properties of the majority of boron-dipyrromethene dyes were found to be located in the visible spectral range. To our knowledge, only one example of such chromophores, namely the Keio Fluors featuring both electron donors and acceptors, reaches fluorescence emissions in the NIR region with high fluorescence quantum yields. ${ }^{9}$ Other approaches were proposed in the literature to reach concomitant NIR fluorescence and high extinction coefficients with boron-dipyrromethene dyes. They were based either on (1) the introduction of electron donating moieties; ${ }^{10,11}$ (2) the extension of the $\pi$-conjugation by the introduction of extensively delocalized groups at the 3-position, ${ }^{12-14}$ or the fusion of aryl moieties, ${ }^{15-18}$ or (3) the stiffening of the borondipyrromethene skeleton. ${ }^{9,19,20}$ Eventually, recent studies have shown that substituting the meso-carbon atom by a nitrogen, giving rise to the so-called aza-boron-dipyrromethene (referred as aza-BODIPY) dyes ( $\mathbf{1}$ and $\mathbf{2}$ on Fig. 1), shifts the photophysical properties to the red with emission at $672 \mathrm{~nm}$ and $687 \mathrm{~nm}$ for $\mathbf{1}$ and 2, respectively. ${ }^{21-24}$ Among those, O'Shea's chromophore $\mathbf{3}$ functionalised with weak electro-donating methoxy groups at the $3 / 5$ positions, which exhibits an emission at $715 \mathrm{~nm}$ with $\Phi=0.36,{ }^{21,22}$ is now considered as the reference in the family and is used as bio-probes for imaging 


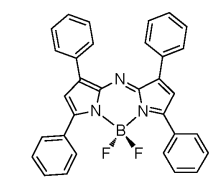

1 $\lambda_{\max }\left(\mathrm{CHCl}_{3}\right)=650 \mathrm{~nm}$ $\lambda_{\text {em }}\left(\mathrm{CHCl}_{3}\right)=672 \mathrm{~nm}$
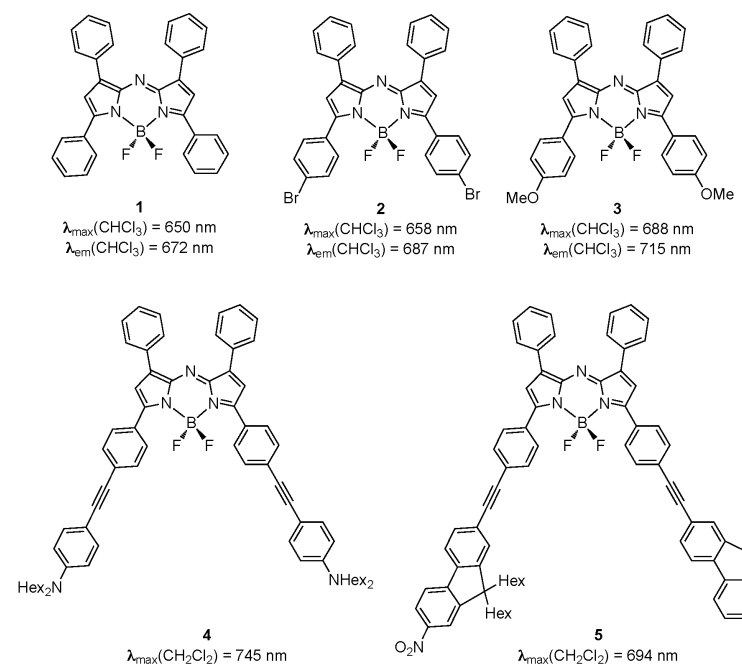
$\begin{aligned} 4 & \\ \lambda_{\max }\left(\mathrm{CH}_{2} \mathrm{Cl}_{2}\right) & =745 \mathrm{~nm} \\ \lambda_{\text {em }}(\text { toluene }) & =827 \mathrm{~nm}\end{aligned}$ $\mathrm{O}_{2}$ $\lambda$ en
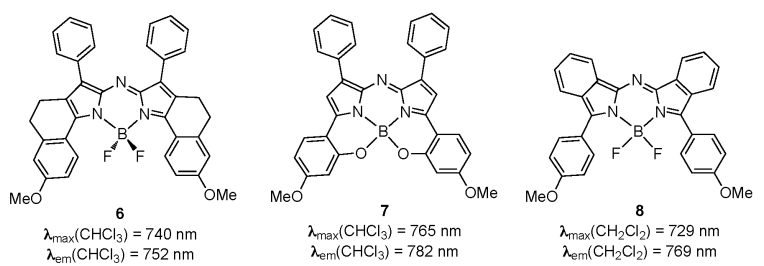

Fig. 1 Representation of typical aza-BODIPY dyes investigated herein.

and sensing applications. ${ }^{25-28}$ Along this line, two of us demonstrated that the extension of the conjugated pathway in the presence (4), ${ }^{29}$ or the absence $(\mathbf{5})^{24}$ of electron-donating fragments strongly enhances the bathochromic shift. Indeed, compound $\mathbf{5}$ exhibits a strong emission at $741 \mathrm{~nm}\left(\Delta \lambda_{\mathrm{em}}=26 \mathrm{~nm}\right.$ w.r.t. 3), whereas 4 exhibits a rather modest emission at $827 \mathrm{~nm}$ in toluene. Details of its photophysical properties will be discussed elsewhere. Moreover, as for boron-dipyrromethenes, NIR bathochromic shifts were obtained upon stiffening of the central skeleton either by annelation $\left(\mathbf{6}, \Delta \lambda_{\mathrm{em}}=36 \mathrm{~nm} \text { wrt. 3) }\right)^{30,31}$ or by formation of intramolecular B-O six members rings $\left(7, \Delta \lambda_{\mathrm{em}}=67 \mathrm{~nm}\right.$ w.r.t. 3). ${ }^{32}$ Unfortunately, this interesting NIR displacement of the spectroscopic properties was accompanied by a significant decrease of the quantum yield efficiency. More recently, Gresser et al. revisited the synthesis of aza-diisoindolmethine dyes and their corresponding boron difluoride. ${ }^{33}$ The systematic investigation of the influence of the substitution on the absorption properties allowed them to reach fluorescence in the range between $723 \mathrm{~nm}$ and $841 \mathrm{~nm}$. With methoxy groups at the $3 / 5$ positions, as in compound 3 , the emission is observed at $769 \mathrm{~nm}\left(\mathbf{8}, \Delta \lambda_{\mathrm{em}}=54 \mathrm{~nm}\right.$ w.r.t. 3).

In the framework of optimising the properties of dyes, and more precisely of the design of NIR chromophores, theoretical simulations are often viewed as efficient tools to help guiding the synthesis towards the most promising derivatives, or, at least, to provide a complementary spectroscopic view ${ }^{34}$ on the measured data. To screen a large number of compounds, it is of interest to use a theory that provides reasonable absolute transition energies as well as accurate auxochromic effects for a moderate computational cost. Time-Dependent Density Functional Theory (TD-DFT), ${ }^{35-37}$ the extension of DFT to electronically excited-states, occupies a key spot in that scene, as it may be regarded as one of the most valuable approaches in terms of the accuracy/effort ratio. Indeed, on the one hand, semi-empirical schemes, such as the popular ZINDO/S, are lightning fast but tend to lack of consistency, general-purpose parameters, and more importantly present difficulty foreseeable errors, ${ }^{38}$ whereas, on the other hand, highly-correlated wavefunction methods (EOM-CC, CAS-PT2, MR-CI...) are extremely trustworthy but are impossible to apply on a extended set of large molecules, such as BODIPY derivatives. For sure, one major problem of (TD-)DFT is the choice of an adequate functional. For absorption spectra, several extended benchmark investigations have been performed during the last decade. ${ }^{3-51}$ Though the set of molecules treated and the selected computational protocol differ from one investigation to the other, some general findings have emerged: (1) pure functionals tend to behave poorly; (2) average errors with the "best" functionals are of the order of $0.2-0.3 \mathrm{eV}$; (3) the deviations tend to be smaller for $n \rightarrow \pi^{*}$ than for $\pi \rightarrow \pi^{*}$ transitions; (4) for local transitions, global hybrids containing ca. $25 \%$ of exact exchange are often adequate; (5) chargetransfer and Rydberg states can be more adequately described with range-separated hybrids or global hybrids presenting a large (ca. 50\%) exact exchange share; (6) excited-state presenting a strong double character cannot be adequately described by the common mono-determinantal implementation of TD-DFT; (7) cyanine-like transitions are difficult to simulate, irrespective of the selected functional as they all yield very similar results for this family of transitions. ${ }^{44,52}$ This latter drawback is not related to the multi-determinantal nature of the problem, ${ }^{53}$ but to a more intricate set of reasons. ${ }^{54}$ There are intense discussions in the literature on ways to resolve this difficulty, ${ }^{54-57}$ but we will concentrate here on the states dominated by a CT nature, so to circumvent these difficulties. To the best of our knowledge, there has been no previous systematic study aiming to propose a computational procedure adequate for aza-BODIPY dyes, though one can find several specific investigations using standard approaches. ${ }^{24,33,58}$ This contribution fills this gap as we have performed basis set and functional benchmarks prior to propose new structures based on the aza-BODIPY core absorbing in the near IR. Such a task is not necessarily "exciting" (and we have tried to stay compact) but is a required preliminary step for a forthcoming accurate design of efficient NIR aza-BODIPY dyes. As we aim to screen a set of compounds, we resort to the vertical approximation of TD-DFT. Of course, a more rigourous approach would be reached by computing the $0-0$ transitions (a task requiring optimising the excited-state geometry and, normally, computing the related vibrational modes using a numerical approach). ${ }^{50}$ For a model system of aza-BODIPY we have performed such calculation to assess the errors related to the vertical approach, but it is clear that $0-0$ calculations are beyond computational reach for the extended set of large molecules investigated herein.

This paper is organised as follows. In Section 2, we briefly summarise our computational approach. In Section 3, the absorption spectra of various molecules based on the aza-BODIPY core are computed with different basis sets and several pure, global, and range-separated hybrids functionals and compared to experimental data. Section 4 is devoted to a better understanding of the origin of the main transitions for the central 
compounds of the study. Finally, novel strategies for NIR aza-BODIPY dyes are explored in Section 5.

\section{Computational details}

All simulations have been achieved with Gaussian09 program, ${ }^{59}$ applying default procedures, integration grids, algorithms and parameters, except for tighten SCF $\left(10^{-8}-10^{-10}\right.$ a.u.) and internal forces $\left(10^{-5}\right.$ a.u.) convergence thresholds. We have adopted a three-step computational strategy that has been found to be adequate for evaluating the spectral properties of organic and inorganic dyes. ${ }^{60} \mathrm{We}$ refer the readers to this publication for general discussion and references. The computational protocol consists of three successive stages: (1) the ground-state geometrical parameters have been calculated at the DFT level, through a force-minimisation process; (2) the vibrational spectrum of each derivatives has been determined analytically at the same level of theory and it has been checked that all structures correspond to true minima of the potential energy surface; (3) the first ten low-lying excitedstates have been calculated within the vertical linear-response TD-DFT approximation. ${ }^{35,36}$ All calculations have been done using the $C_{2}$ symmetry (except for compounds 5 and 8 ). As stated in the Introduction, there is, to the best of our knowledge, no previous TD-DFT benchmarks dedicated to the BODIPY series of dyes. For this reason, we have carried out both basis set and functional assessments (see Section 3). The latter used a wide panel of DFT functionals, from GGA to recent range-separated hybrids: $\mathrm{PBE},{ }^{61} \mathrm{TPSS}^{62}{ }^{6 \mathrm{TPSS}},{ }^{63}$ O3LYP ${ }^{64}$ B3LYP ${ }^{65,66}$ PBE0,${ }^{67,68}$ BMK, ${ }^{69}$ M05-2X, ${ }^{70}$ LC-PBE, ${ }^{71,72}$ LC- $\omega$ PBE, ${ }^{73,74}$ CAM-B3LYP $,{ }^{75} \omega \mathrm{B}^{7},{ }^{76} \omega \mathrm{B} 97 \mathrm{X}^{76}$ and $\omega \mathrm{B} 97 \mathrm{XD} .{ }^{77}$ Except when noted, we systematically (all steps) include a modeling of bulk solvent effects through the Polarisable Continuum Model (PCM) ${ }^{78}$ using a linear-response non-equilibrium approach for the TD-DFT step. ${ }^{79,80}$ For the $0-0$ calculations, the geometry optimisations of the excitedstate and the related (numerical) frequency calculations have been performed using the (default) equilibrium approach adequate for force minimizations at the TD-DFT level. In this contribution, the contour threshold selected to represent the molecular orbitals was systematically set to 0.030 a.u.

\section{Benchmarks}

\subsection{Basis sets}

First, we have evaluated the impact of the selected atomic basis set (BS) for the TD-DFT step, as excited-state properties are often significantly BS-dependent. To this end, we have used the simplest model ( 1 in Fig. 1) and one global hybrid, PBE0, as BS effects should be relatively constant within one chromophoric family. The obtained results are listed in Table S1, in ESI. $\dagger$ Three principal transitions are predicted, and the two latter $\left(\lambda_{2}\right.$ and $\lambda_{3}$ in Table S1, ESI $\left.\dagger\right)$ are almost unaffected by the nature of the BS. Indeed, provided polarisation functions are included, their calculated wavelengths (oscillator strengths) vary by $2 \mathrm{~nm}(0.02)$, at most. For the $\lambda_{\max }$, the major focus of the present article, the largest BS, namely $6-311++\mathrm{G}(2 \mathrm{df}, 2 \mathrm{p})$, yields $600 \mathrm{~nm}(f=0.86)$. Diffuse orbitals are clearly required to approach
BS convergence for this band, e.g. 6-31G(d,p) and 6-311G(d,p) imply a non-negligible error of $c a$. $10 \mathrm{~nm}$. All triple- $\zeta$ diffusecontaining BS are very close to the $6-311++\mathrm{G}(2 \mathrm{df}, 2 \mathrm{p})$ reference, though it turns out that the addition of a second set of $d$ orbitals on the non-hydrogen atoms still induces a small shift. Therefore, as we aim to benchmark functional with a BS-error free model, we have selected $6-311+\mathrm{G}(2 \mathrm{~d}, \mathrm{p})$.

Next, we have investigated the impact of BS on the geometrical parameters. The results are collated in ESI $\dagger$ (Tables S2-S4). The two largest BS, namely cc-pVTZ and $6-311++\mathrm{G}(2 \mathrm{~d}, 2 \mathrm{p})$, provide extremely similar values: the bond lengths are within $0.001 \AA$ of each other but for the boron-nitrogen distance (0.004 $\AA$ variation), the valence angles only differ by $0.1^{\circ}$, and the transition wavelengths show $1 \mathrm{~nm}$ variation. Therefore, these two BS provide structural parameters very close to the BS limit at the DFT level. In Table S3 (ESI $\dagger$ ), one notes that the FBF angle tends to decrease by $c a .0 .5^{\circ}$ when going from double- $\zeta$ to triple- $\zeta$ BS. A similar behaviour appears for the $\lambda_{\text {max }}: 594 \mathrm{~nm}$ with cc-pVTZ but $602 \mathrm{~nm}$ with 6-31G(2d,2p). By restricting our analysis to the triple- $\zeta$ set, we note that a second set of $d$ polarization function is necessary to take the inner track to BS convergence. On the contrary, diffuse orbitals have a minor impact once a $2 d$-BS is applied, as they only perturb the bond lengths (transition energies) by $0.001-0.002 \AA(1-2 \mathrm{~nm})$.

Consequently, we have systematically applied a $6-311+\mathrm{G}(2 \mathrm{~d}, \mathrm{p}) / /$ $6-311 \mathrm{G}(2 \mathrm{~d}, \mathrm{p})$ combination for the excited-state//ground-state properties in the following. Of course, a first reasonable approximation could have been obtained with a $6-31+\mathrm{G}(\mathrm{d}) / / 6-31 \mathrm{G}(\mathrm{d})$ blend, but, as we wish to benchmark functionals we strive to avoid any significant BS bias in our assessment.

\subsection{Functionals}

Pinpointing an adequate functional is more difficult than choosing a BS, as one cannot refer to a "functional limit" nor highly-accurate wavefunction results when molecules are too large to allow coupled-cluster (or alike) force minimizations. Consequently, one has to resort to experimental data. For the structural parameters, we have chosen to compare gasphase DFT/6-311G(2d,p) estimates to available XRD data for both $2^{24}$ and $3^{21}$ derivatives. The computed mean absolute errors (MAE) for the bond lengths and valence angles of the chromogen can be found in Table 1 whereas Tables S5 and S6 $(\mathrm{ESI} \dagger)$ provide a complete list of parameters. Experimentally, the structures are not perfectly of $C_{2}$ symmetry due to the influence of the solid-state effects, and we have used the average measured data for the two halves of the molecules in our comparisons. From Table 1, PBE0 clearly emerges as the most adequate functional as it yields the smallest MAE for the bond lengths of both compounds, is on the experimental spot for the $\mathrm{B}-\mathrm{N}$ bond length and is also very accurate for the valence angles (average deviations smaller than $1.0^{\circ}$ ). The only significant error of PBE0 is the B-F bond length of the methoxy derivative (1.384 $\AA$ instead of $1.371 \AA$ ), a discrepancy that can only be significantly reduced with LC-PBE, a functional performing poorly on average. PBE0 has therefore been selected for all geometry optimisations. In short, pure functionals (e.g. PBE) and range-separated hybrids relying on a large attenuation parameter (e.g. LC-PBE) are rather unsatisfying, but most hybrids yield 
Table 1 Mean absolute errors (DFT versus XRD) computed for selected bond lengths (in $\AA$ ) and valence angles (in degrees) for two structures. The reference experimental values have been taken from ref. 21 and 24 for the bromo (2) and methoxy (3) derivatives, respectively. See text for more details and Tables S5 and S6 for full list of values

\begin{tabular}{llllll}
\hline & \multicolumn{2}{l}{ Bond lengths } & & \multicolumn{2}{c}{ Valence angles } \\
\cline { 2 - 3 } Functional & $\mathbf{2}$ & $\mathbf{3}$ & & $\mathbf{2}$ & $\mathbf{3}$ \\
\hline PBE & 0.009 & 0.013 & 0.8 & 0.5 \\
TPSS & 0.008 & 0.012 & 0.7 & 0.4 \\
TPSSh & 0.005 & 0.008 & 0.7 & 0.4 \\
O3LYP & 0.007 & 0.009 & 1.0 & 0.7 \\
B3LYP & 0.006 & 0.008 & 0.7 & 0.5 \\
PBE0 & 0.005 & 0.007 & 0.8 & 0.5 \\
BMK & 0.009 & 0.009 & 0.8 & 0.6 \\
M05-2X & 0.007 & 0.009 & 0.9 & 0.4 \\
LC-PBE & 0.017 & 0.016 & 0.9 & 0.6 \\
LC- $\omega$ PBE & 0.009 & 0.011 & 1.0 & 0.8 \\
CAM-B3LYP & 0.005 & 0.008 & 0.8 & 0.6 \\
$\omega$ B97 & 0.010 & 0.010 & 0.9 & 0.5 \\
$\omega$ B97X & 0.007 & 0.010 & 0.9 & 0.5 \\
$\omega$ B97XD & 0.009 & 0.009 & 0.9 & 0.5 \\
\hline
\end{tabular}

reasonable estimates with MAE smaller than $0.010 \AA$ and $1.0^{\circ}$ for both molecules.

For the spectral properties, we have initially focussed on two structures in which the $\lambda_{\max }$ possesses a strong CT character, namely 4 (push) ( $^{29}$ and 5 (pull) ${ }^{24}$ of Fig. 1, as we aim to design near-IR dyes. These systems differ not only by their donor/ acceptor character but also by the length and nature of the conjugated path linking the electro-active groups to the central chromogenic core. In both cases, the calculations used methyl instead of hexyl side chains. The $\lambda_{\max }$ are collated in Table 2 but a more complete description of all bands may be found in Tables S7 and S8 (ESI $\dagger$ ). As stated in the Introduction, we have performed pragmatic vertical benchmarks. The assignment of all bands is relatively straightforward for $\mathbf{5}$ but is more difficult for 4 . For the latter, we have first pinpointed the $\lambda_{\max }$ and the very intense band in the near-UV domain (see experimental spectra in ref. 29) and next positioned the in-between transitions (see Table S7, ESI $\dagger$ ). From Table 2, we note that pure functionals are completely inadequate: they predict much too small transition energies, as expected. Range-separated hybrids undershoot the $\lambda_{\max }$, but more importantly underestimate the 4-5 difference and, at the exception of CAM-B3LYP, they do not predict any significant transitions in the 400-600 $\mathrm{nm}$ domain for 4, in obvious contradiction with experiment. Global hybrids including a $10-30 \%$ of exact exchange are accurate for 5 but less satisfying for $\mathbf{4}$ as they yield a too large $\lambda_{\max }$ as well as extra transitions that remain unseen experimentally (Table S7, ESI $\dagger$ ). Eventually BMK seems to be a good compromise: it slightly underestimates the wavelengths but recovers reasonable position and intensities for the different bands, as well as an accurate auxochromic shift. Indeed, it predicts a 4-5 hypsochromic shift of $-64 \mathrm{~nm}$ instead of the $-47 \mathrm{~nm}$ measured value, whereas B3LYP provides $-172 \mathrm{~nm}$, an exaggerated value typical of CT states, and $\omega \mathrm{B} 97$ gives $-16 \mathrm{~nm}$. In summary, our computational protocol in the following is PCM-TD-BMK/6-311+G(2d,p)// PCM-PBE0/6-311G(2d,p).

In order to provide a complete information, we have also benchmarked the same set of functionals for three dyes with
Table 2 Comparison between theoretical and experimental $\lambda_{\max }$ in $\mathrm{nm}$. Oscillator strengths are listed between brackets. The theoretical values are evaluated at the $\mathrm{PCM}\left(\mathrm{CH}_{2} \mathrm{Cl}_{2}\right)$-TD-DFT/6-311+G(2d,p)// $\operatorname{PCM}\left(\mathrm{CH}_{2} \mathrm{Cl}_{2}\right)-\mathrm{PBE} 0 / 6-311 \mathrm{G}(2 \mathrm{~d}, \mathrm{p})$ level of approximation. The experimental values have been taken from ref. 24 and 29 , for $\mathbf{4}$ and $\mathbf{5}$, respectively

\begin{tabular}{llr}
\hline Functional & $\mathbf{4}$ & $\mathbf{5}$ \\
\hline PBE & $1267(0.54)$ & $852(0.92)$ \\
TPSS & $1212(0.55)$ & $829(0.95)$ \\
TPSSh & $1021(0.62)$ & $750(1.09)$ \\
O3LYP & $1011(0.62)$ & $751(1.09)$ \\
B3LYP & $885(0.76)$ & $713(1.18)$ \\
PBE0 & $834(0.85)$ & $690(1.22)$ \\
M06 & $810(0.91)$ & $701(1.20)$ \\
BMK & $711(1.14)$ & $647(1.27)$ \\
M05-2X & $688(1.22)$ & $649(1.24)$ \\
LC-PBE & $672(1.19)$ & $655(1.17)$ \\
LC- $\omega$ PBE & $669(1.18)$ & $652(1.16)$ \\
CAM-B3LYP & $679(1.21)$ & $648(1.22)$ \\
$\omega$ B97 & $673(1.18)$ & $657(1.16)$ \\
$\omega$ B97X & $665(1.19)$ & $647(1.18)$ \\
$\omega$ B97XD & $662(1.21)$ & $641(1.20)$ \\
Exp & 741 & 694 \\
\hline
\end{tabular}

zero (1), trifling (2) or small (3) CT character as well as rigidified dyes, 6, 7 and $\mathbf{8}$ (Table 3), that are structures dominated by a cyanine-like response. For $\mathbf{1}$, the range of variation of the computed $\lambda_{\max }$ is small $(0.16 \mathrm{eV}$ : from $630 \mathrm{~nm}$ with PBE to $583 \mathrm{~nm}$ with BMK, whereas it was $c a .1 \mathrm{eV}$ for 4) and the computed transition energies are always too large (exp: $650 \mathrm{~nm}){ }^{22}$ two hallmarks of cyanine states simulated by conventional TD-DFT approaches. ${ }^{47,52-54}$ When going to the bromo (2) or methoxy (3) derivatives, the range of variation slightly increases but the cyanine-like character still dominates the response. The same holds for $\mathbf{6 , 7}$ and $\mathbf{8}$ with almost constant values when modifying the functionals $(0.14 \mathrm{eV}, 0.17 \mathrm{eV}$ and $0.18 \mathrm{eV}$, respectively), which indicates that rigidifying the chromophore increases the $\lambda_{\max }$ (an effect qualitatively restored by TD-DFT) but does not imply a modification of the nature of the excitedstate. In the present case, the cyanine nature of the states can be indirectly pinpointed as the evolution of the wavelength is not a smooth function of the exact exchange ratio (in global hybrids) nor of the attenuation parameter (in range-separated hybrid). Therefore, standard vertical TD-DFT should not be advocated in these cases. For all eight dyes of Fig. 1, irrespective of the nature of the excited-state, BMK provides too small $\lambda_{\max }$ with errors in the 30-70 $\mathrm{nm}$ range. Therefore, the values predicted with BMK for unknown dyes of the same family can be considered as reasonable lower bounds of the experimental values. In addition, selecting $\mathbf{1}$ as reference BMK yields auxochromic shifts of $+8 \mathrm{~nm}(\mathbf{2}),+39 \mathrm{~nm}(\mathbf{3}),+71 \mathrm{~nm}(\mathbf{6}),+76 \mathrm{~nm}(7)$ and $+83 \mathrm{~nm}(8)$ that are certainly satisfactory as experimental values are $+8 \mathrm{~nm},+37 \mathrm{~nm},+90 \mathrm{~nm},+115 \mathrm{~nm}$ and $+79 \mathrm{~nm}$, respectively. ${ }^{21,22,24,31-33}$ Sure, the variations w.r.t. 4 and 5 may appear more disappointing and we underline that we recommend the BMK functional only for comparisons of structures displaying similar states (CT or cyanine). For this reason we focus on CT structures in the following.

Eventually, though we aim to provide reasonable chemical (i.e. relative evolution upon substitution) $\lambda_{\max }$, rather than absolute raw values, let us investigate the origin of the quite large errors obtained with the selected TD-DFT model. These discrepancies 
Table 3 Comparison between theoretical and experimental $\lambda_{\max }$ in $\mathrm{nm}$. Oscillator strengths are listed between brackets. The theoretical values are evaluated at the PCM-TD-DFT/6-311+G(2d,p)//PCM-PBE0/6-311G(2d,p) level of approximation. The experimental values have been taken from the literature and use the following media: $\mathrm{CHCl}_{3}$ for $\mathbf{1}^{22} \mathbf{6}^{31}$ and $7,{ }^{32} \mathrm{CH}_{2} \mathrm{Cl}_{2}$ for $\mathbf{2}^{24} \mathbf{3}^{21}$ and $\mathbf{8}^{33}$

\begin{tabular}{|c|c|c|c|c|c|c|}
\hline Functional & 1 & 2 & 3 & 6 & 7 & 8 \\
\hline PBE & $630(0.72)$ & $655(0.80)$ & $703(0.72)$ & $706(0.94)$ & $726(0.56)$ & $738(0.90)$ \\
\hline TPSS & $619(0.76)$ & $640(0.83)$ & $687(0.75)$ & $691(0.98)$ & $709(0.56)$ & $723(0.93)$ \\
\hline TPSSh & $604(0.82)$ & $621(0.86)$ & $661(0.80)$ & $677(0.98)$ & $691(0.56)$ & $697(0.86)$ \\
\hline O3LYP & $609(0.81)$ & $626(0.86)$ & $666(0.79)$ & $683(0.96)$ & $697(0.55)$ & $702(0.84)$ \\
\hline B3LYP & $603(0.84)$ & $616(0.89)$ & $652(0.83)$ & $675(0.99)$ & $685(0.58)$ & $699(0.80)$ \\
\hline PBE0 & $596(0.87)$ & $608(0.91)$ & $643(0.86)$ & $668(1.01)$ & $677(0.60)$ & $687(1.03)$ \\
\hline M06 & $615(0.85)$ & $628(0.89)$ & $659(0.84)$ & $687(0.99)$ & $693(0.59)$ & $703(1.06)$ \\
\hline BMK & $583(0.91)$ & $591(0.96)$ & $622(0.91)$ & $653(1.06)$ & $659(0.64)$ & $666(1.17)$ \\
\hline M05-2X & $596(0.90)$ & $603(0.95)$ & $632(0.91)$ & $668(1.05)$ & $671(0.65)$ & $677(1.18)$ \\
\hline LC-PBE & $621(0.93)$ & $625(0.97)$ & $635(0.95)$ & $703(1.09)$ & $699(0.69)$ & $713(1.23)$ \\
\hline LC- $\omega$ PBE & $618(0.91)$ & $622(0.95)$ & $651(0.93)$ & $697(1.07)$ & $697(0.67)$ & $709(1.21)$ \\
\hline CAM-B3LYP & $599(0.90)$ & $605(0.94)$ & $634(0.91)$ & $671(1.05)$ & $675(0.64)$ & $682(1.17)$ \\
\hline$\omega \mathrm{B} 97$ & $622(0.91)$ & $626(0.95)$ & $654(0.93)$ & $701(1.07)$ & $701(0.67)$ & $712(1.21)$ \\
\hline$\omega \mathrm{B} 97 \mathrm{X}$ & $611(0.91)$ & $615(0.96)$ & $643(0.93)$ & $687(1.07)$ & $688(0.66)$ & $698(1.20)$ \\
\hline$\omega \mathrm{B} 97 \mathrm{XD}$ & $599(0.91)$ & $604(0.95)$ & $633(0.92)$ & $672(1.06)$ & $675(0.64)$ & $684(1.17)$ \\
\hline Exp & 650 & 658 & 687 & 740 & 765 & 729 \\
\hline
\end{tabular}

may be ascribed to the vertical approach. Indeed, using the $\operatorname{PCM}\left(\mathrm{CHCl}_{3}\right)-\mathrm{PBE} 0 / 6-311 \mathrm{G}(2 \mathrm{~d}, \mathrm{p})$ we have computed a theoretical estimate of the $0-0$ spectra for the chromophoric core. This model is presented in Fig. 2 together with the bond lengths obtained at the DFT and TD-DFT level, indicating a strong elongation of the central $\mathrm{C}-\mathrm{N}$ bonds of the central ring and a contraction of the $\mathrm{B}-\mathrm{N}$ bonds upon photon absorption. For the records, it turned out that both the $S_{0}$ and $S_{1}$ structures belong to the $C_{2 v}$ point group. For this model system, PCM-PBE0 provides a vertical transition energy of $2.77 \mathrm{eV}$, whereas the corresponding $0-0$ values (including the $0.10 \mathrm{eV}$ ZPVE correction) are significantly smaller: $2.48 \mathrm{eV}$. Given the fact that experimentally, the $\lambda_{\max }$ and $0-0$ point are extremely close, it is therefore clear that the underestimation of the absorption wavelength by TD-DFT is partly ascribable to the vertical approximation, that remains, once more, the only computationally-tractable approach for a large set of dyes.

\section{Absorption spectra of 4 and 5: a closer look}

Before trying to optimize the absorption wavelength of substituted dyes, we analyse the nature of the main transitions of the pull and push dyes by determining the topology of the molecular orbitals implied in these transitions. The frontier orbitals computed for $\mathbf{4}$ and $\mathbf{5}$ can be found in Fig. 3 and 4, respectively. For 4 (see Table S7, ESI $\dagger$ ), the $\lambda_{\max }$ at $711 \mathrm{~nm}$ is dominated by an HOMO to LUMO electronic promotion but also contains a small HOMO -2 to LUMO component. It is

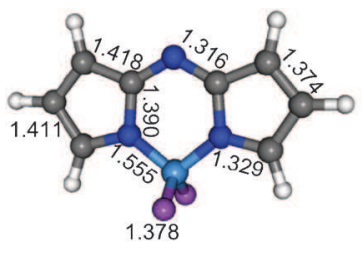

$S_{0}$

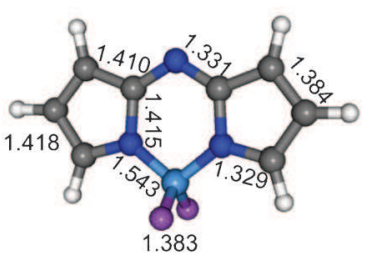

$S_{1}$
Fig. 2 Ground (left) and excited state (right) geometries for a model chromophore obtained at the PCM-(TD)-PBE0/6-311G(2d,p) level. Main bond lengths are indicated $(\AA)$. See text for more details. obvious from Fig. 3 that this absorption therefore corresponds to a charge-transfer from the amino arms to the central azaBODIPY core, though there is still a significant overlap between the occupied and virtual orbitals (minor cyanine-like contribution). On the contrary, the second band (at $562 \mathrm{~nm}$ ) implies an HOMO - 1 to LUMO transition and is a clear-cut CT state. The less intense $411 \mathrm{~nm}$ band is dominated by a HOMO - 4 to LUMO component, the former being localised on the top phenyl rings. Eventually, the two largest contributions in the very intense band at $343 \mathrm{~nm}$ are HOMO to LUMO + 1 and HOMO -1 to LUMO + 1, that is, absorption of a UV photon mainly induces electronic redistribution on the long arms, but has no major effect on the aza-BODIPY chromogen. In 5 (see Table S8, ESI $\dagger$ ), the $647 \mathrm{~nm} \lambda_{\max }$ is almost a pure HOMO to LUMO band with a marked cyanine-like character. Contrary to its amino counterpart, the CT character is rather limited, as the HOMO is not localized on the nitro-fluorene moiety. As the LUMOs of $\mathbf{4}$ and $\mathbf{5}$ present alike topologies, we can infer that the aza-BODIPY core acts as a strong accepting group, so that the addition of an extra electro-accepting moiety is not ideal to induce large CT. The 448 and $379 \mathrm{~nm}$ bands of 5 imply a complex orbital blend but are dominated by an $\mathrm{HOMO}-1$ to LUMO and an HOMO to LUMO + 1 contribution, respectively. In other words, the $448 \mathrm{~nm}$ absorption implies a CT from the fluorene arms towards the aza-BODIPY core, whereas the $379 \mathrm{~nm}$ presents the opposite character. Therefore, the chemical nature of the bands of these two dyes strongly differs, though their optical spectra are relatively comparable.

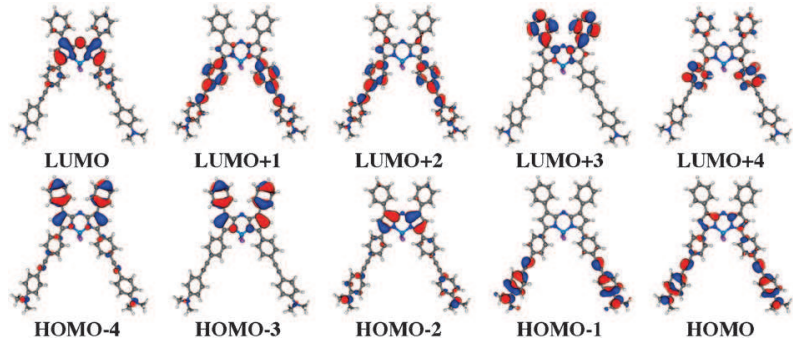

Fig. 3 Five frontier occupied and virtual orbitals for $\mathbf{4}$ obtained at the $\mathrm{BMK} / 6-311+\mathrm{G}(2 \mathrm{~d}, \mathrm{p})$ level using a contour threshold of 0.03 a.u. 


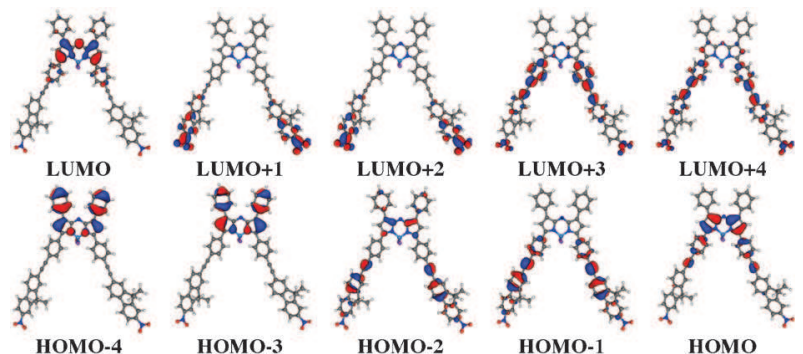

Fig. 4 Frontier orbitals of 5. See caption of Fig. 3.

\section{Designing new aza-BODIPY models for NIR applications}

With the design of new aza-BODIPY molecules for NIR applications in mind, $\lambda_{\max }$ have been calculated at the $\mathrm{PCM}\left(\mathrm{CH}_{2} \mathrm{Cl}_{2}\right)$ TD-BMK/6-311+G(2d,p)//PCM( $\left.\mathrm{CH}_{2} \mathrm{Cl}_{2}\right)$-PBE0/6-311G(2d,p) level for a series of hypothetic molecules, following several chemically-sound strategies. First, the donor and acceptor moieties which are at the origin of the specificities of complexes $\mathbf{4}$ and $\mathbf{5}$ were "grafted" to systems 6-8 instead of the original methoxy groups, giving rise to six new model molecules (9-14, see Fig. 5). In parallel, by mixing the structural peculiarities (annelation, intramolecular 6-membered $\mathrm{B}-\mathrm{O}$ ring, ...) of these complexes, six additional architectures were proposed (15-20, see Fig. 5). The calculated $\lambda_{\max }$ for this series of molecules are collated in Table 4. As expected, the behaviour of these proposed complexes mimics the trend observed between $\mathbf{4}$ and $\mathbf{5}$, with a shift to the red when replacing the acceptor group by the donor moiety. Moreover, whilst the bathochromic displacements w.r.t. the methoxy-molecules are rather limited for compounds bearing the accepting moieties $(+45,+31$, and $+22 \mathrm{~nm}$ for $\mathbf{1 0}, \mathbf{1 2}$, and $\mathbf{1 4}$, respectively), they are appreciably larger for dyes with the donor groups $(+94$, +76 , and $+49 \mathrm{~nm}$ for $\mathbf{9}, \mathbf{1 1}$, and $\mathbf{1 3}$, respectively). This can be partially explained by the different nature of the lowest-energy transition which is of cyanine-like character in the methoxy derivatives and is dominated by CT contributions in the D-functionalised hypothetic molecules. At this stage, it is already clear from these calculations that the proposed donor-based
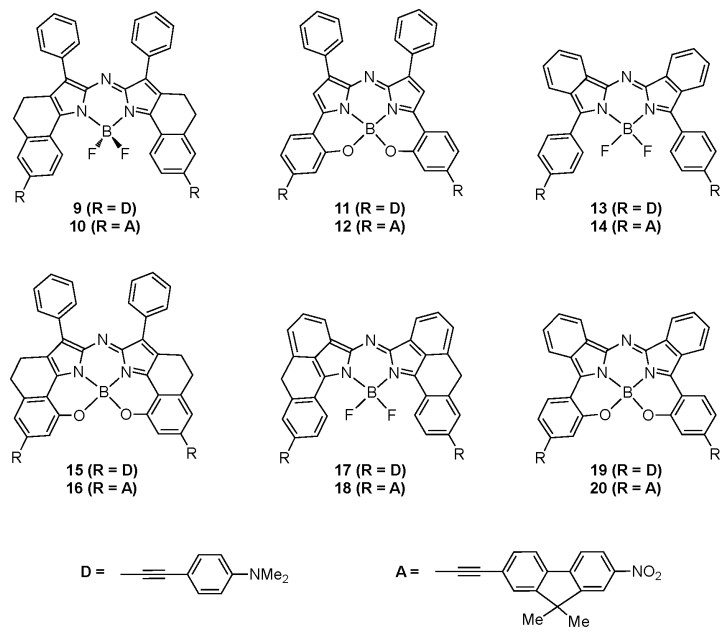

Fig. 5 Representation of hypothetic aza-BODIPY dyes.
Table 4 Calculated $\lambda_{\max }$ (in $\mathrm{nm}$ ) for the hypothetic molecules 9-20 (see Fig. 5). Oscillator strengths are listed between brackets. The theoretical values are evaluated at the $\mathrm{PCM}\left(\mathrm{CH}_{2} \mathrm{Cl}_{2}\right)$-TD-BMK/ 6-311+G(2d,p)//PCM(CH$\left.{ }_{2} \mathrm{Cl}_{2}\right)-\mathrm{PBE} 0 / 6-311 \mathrm{G}(2 \mathrm{~d}, \mathrm{p})$ level of approximation

\begin{tabular}{llll}
\hline & $\lambda_{\max }$ & & $\lambda_{\max }$ \\
\hline $\mathbf{9}$ & $747(1.37)$ & $\mathbf{1 0}$ & $698(1.48)$ \\
$\mathbf{1 1}$ & $735(0.66)$ & $\mathbf{1 2}$ & $690(0.75)$ \\
$\mathbf{1 3}$ & $715(1.13)$ & $\mathbf{1 4}$ & $688(1.18)$ \\
$\mathbf{1 5}$ & $761(0.90)$ & $\mathbf{1 6}$ & $728(0.97)$ \\
$\mathbf{1 7}$ & $797(1.47)$ & $\mathbf{1 8}$ & $786(1.50)$ \\
$\mathbf{1 9}$ & $789(0.72)$ & $\mathbf{2 0}$ & $769(0.76)$ \\
\hline
\end{tabular}

molecule 9 is an interesting target for potential NIR applications with the calculated $\lambda_{\max }$ at approx. $750 \mathrm{~nm}$. Since BMK calculations always underestimate the experimental value (by 30 to $70 \mathrm{~nm}$, see above), it is reasonable to predict an experimental absorption band at $c a .800 \mathrm{~nm}$ for $\mathbf{9}$. On the other hand, the $\lambda_{\max }$ values calculated for 15-20 underline the complementarity of the various stiffening technics for obtaining larger bathochromic shifts since all computed $\lambda_{\max }$ substantially exceeds those of the parent complexes. In particular, the strongest red-shift is reached for $17\left(\lambda_{\max }=797 \mathrm{~nm}\right)$ and $18\left(\lambda_{\max }=786 \mathrm{~nm}\right)$ for which the annelation strategy proposed by Zhao and Carreira ${ }^{31}$ is applied to the benzannulated azaBODIPY core. Keeping in mind the previously characterised hypsochromic behaviour of the BMK calculations, experimental absorption transitions flirting with $850 \mathrm{~nm}$ could be expected.

Alternatively, we explored the effect on the lowest energy transition of various ligand substitutions $(\mathbf{X}=\mathrm{F}, \mathrm{Cl}, \mathrm{Br}, \mathrm{I}$, $\mathrm{CN}, \mathrm{NO}_{2}, \mathrm{Me}, \mathrm{NH}_{2}$ and ethynyl) at the 2 and 6 positions (based on the usual notation, see for instance ref. 24) of the aza-BODIPY core of complexes $\mathbf{4}$ and $\mathbf{5}(\mathbf{4}-\mathbf{X}$ and $\mathbf{5}-\mathbf{X}$, see Fig. 6 and Table 5). ${ }^{81}$ The longest absorption wavelength is reached for $4-\mathrm{NO}_{2}$, which is obviously a strong push $\left(\mathrm{NMe}_{2}\right.$ tails) and pull (aza-BODIPY core and nitro groups) compound. Nevertheless, adding a donor group, namely $\mathrm{NH}_{2}$, induces a bathochromic shift not only for $\mathbf{5}-\mathbf{N H}_{2}$ (as expected by CT effects), but also for $\mathbf{4}-\mathbf{N H}_{2}$ indicating that pushing electrons from all sides of the molecule to the central chromophore may be a valid strategy to obtain near-IR dyes. On the contrary, poorly electro-active side groups, e.g. methyl, ethynyl, bromine. . have a tendency to yield hypsochromic shifts of the $\lambda_{\max }$. In short, one should use strong but compact electroactive groups at $\mathbf{X}$, acceptors being able to deliver the largest bathochromic shifts.

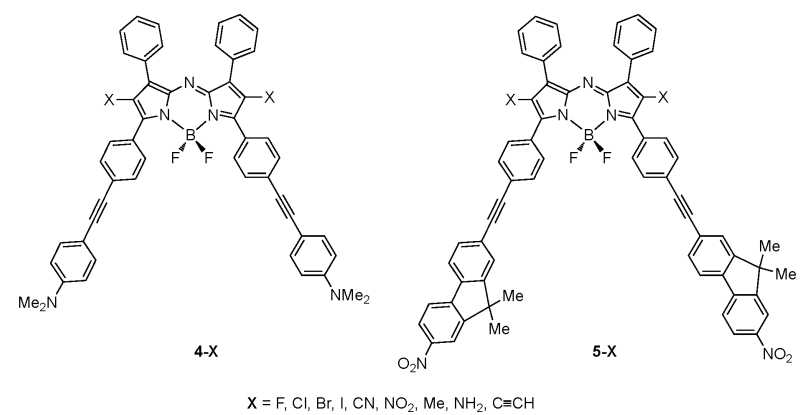

Fig. 6 Representation of hypothetic aza-BODIPY dyes investigated herein. 
Table 5 Calculated $\lambda_{\max }$ (in $\mathrm{nm}$ ) for the hypothetic molecules $\mathbf{4}-\mathbf{X}$ and 5-X with $\mathbf{X}=\mathrm{F}, \mathrm{Cl}, \mathrm{Br}, \mathrm{I}, \mathrm{CN}, \mathrm{NO}_{2}, \mathrm{Me}, \mathrm{NH}_{2}$ and ethynyl (see Fig. 6). Oscillator strengths are listed between brackets. The theoretical values are evaluated at the $\operatorname{PCM}\left(\mathrm{CH}_{2} \mathrm{Cl}_{2}\right)-\mathrm{TD}-\mathrm{BMK} / 6-311+\mathrm{G}(2 \mathrm{~d}, \mathrm{p}) / /$ $\operatorname{PCM}\left(\mathrm{CH}_{2} \mathrm{Cl}_{2}\right)-\mathrm{PBE} 0 / 6-311 \mathrm{G}(2 \mathrm{~d}, \mathrm{p})$ level of approximation

\begin{tabular}{lll}
\hline $\mathrm{X}$ & $\mathbf{4 - X}$ & $\mathbf{5}-\mathbf{X}$ \\
\hline $\mathrm{H}$ & $711(1.14)$ & $647(1.27)$ \\
$\mathrm{F}$ & $752(1.02)$ & $663(1.19)$ \\
$\mathrm{Cl}$ & $697(0.77)$ & $608(1.08)$ \\
$\mathrm{Br}$ & $686(0.74)$ & $600(1.08)$ \\
$\mathrm{I}$ & $658(0.76)$ & $587(1.07)$ \\
$\mathrm{CN}$ & $776(0.72)$ & $631(1.14)$ \\
$\mathrm{NO}_{2}$ & $800(0.57)$ & $626(0.96)$ \\
$\mathrm{Me}_{\mathrm{NH}}$ & $641(0.91)$ & $588(1.10)$ \\
$\mathrm{C} \equiv \mathrm{CH}$ & $755(0.84)$ & $729(0.77)$ \\
\hline
\end{tabular}

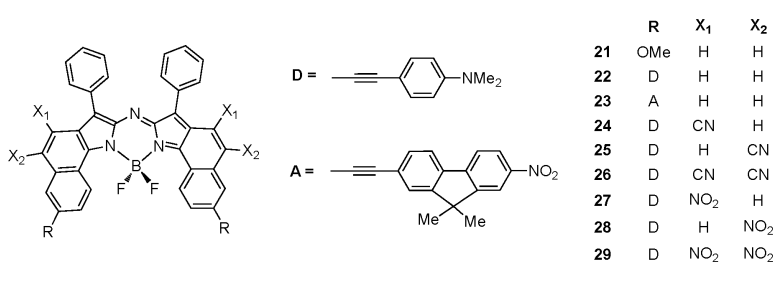

Fig. 7 Representation of hypothetic aza-BODIPY dyes investigated herein.

In our quest to design highly NIR aza-BODIPY chromophores, we finally tested a last strategy consisting in (1) replacing the dimethylene annelation (such as in $\mathbf{6}$, for instance) by $\mathrm{C}_{\mathrm{sp}^{2}}$ ethenyl bridge and (2) modulating the electronic density on this fused aromatic ring by varying the nature of its substituents $\left(\mathrm{X}_{1}, \mathrm{X}_{2}=\mathrm{H}, \mathrm{CN}, \mathrm{NO}_{2}\right)$ as shown in Fig. 7 (model compounds 21 to 29). These modifications are expected to combine a strongly rigid and highly-planar aza-BODIPY core with the large bathochromic effects induced by the $\mathrm{CN}$ and $\mathrm{NO}_{2}$ groups as already observed in $\mathbf{4}-\mathbf{C N}$ and $4-\mathbf{N O}_{2}$. Calculated $\lambda_{\max }$ are listed in Table 6. Comparing computed data obtained for $\mathbf{2 1}$ and $\mathbf{6}$ illustrates the rather trifling effect of an enhanced rigidification of the molecule with a small red shift (665 vs. $653 \mathrm{~nm}$ ). A similar conclusion can be drawn for the molecules bearing the donor and acceptor moieties (22 and 23 w.r.t. 9 and 10, respectively). Nevertheless, let us note that the calculated red shift originating in the improved rigidity reaches almost $60 \mathrm{~nm}$ for the donor-type model ( $806 \mathrm{~nm}$ for 22 vs. $747 \mathrm{~nm}$ for 9). This red shift is strongly accentuated by the presence of either $\mathrm{CN}$ or $\mathrm{NO}_{2}$ auxochromes, irrespective of

Table 6 Calculated $\lambda_{\max }$ (in $\mathrm{nm}$ ) for the hypothetic molecules 21-29 (see Fig. 7). Oscillator strengths are listed between brackets. See caption of Fig. 5 for more details

\begin{tabular}{ll}
\hline & $\lambda_{\max }$ \\
\hline $\mathbf{2 1}$ & $665(1.16)$ \\
$\mathbf{2 2}$ & $806(1.32)$ \\
$\mathbf{2 3}$ & $709(1.57)$ \\
$\mathbf{2 4}$ & $865(1.21)$ \\
$\mathbf{2 5}$ & $874(1.21)$ \\
$\mathbf{2 6}$ & $975(1.07)$ \\
$\mathbf{2 7}$ & $884(1.13)$ \\
$\mathbf{2 9}$ & $895(1.11)$ \\
\hline
\end{tabular}

their positions (see Fig. 7). If both $X_{1}$ and $X_{2}$ positions are occupied (26 and 29), a subsequent $100 \mathrm{~nm}$ shift is observed w.r.t. the singly coordinated molecules with $\lambda_{\max }$ values around $1000 \mathrm{~nm}$. The interest to experimentally obtain such derivatives is therefore quite obvious, despite the difficulty to achieve such syntheses.

\section{Conclusions}

Benchmark calculations aiming at identifying an efficient computational strategy (adequate selection of basis sets and functionals) in the framework of TD-DFT simulations of absorption properties of aza-boron-dipyrromethene dyes have been performed. A large panel of pure functionals, global hybrids, and long-range-corrected hybrids have been tested and results have been compared with experimental data. It appears that an efficient computational protocol consists in the use of the BMK functional for the vertical TD-DFT procedure on top of PBE0 optimized geometries. Nevertheless, it should be noted that the effects related to geometric relaxation of the excited-state are not negligible, so that such a protocol is suited for a quick screening of a large series of aza-BODIPY compounds, but would have to be adapted if $0-0$ calculations including vibrational corrections become computationallytractable for these large dyes.

Using this validated recipe, the design of new potential azaBODIPY molecules for NIR applications has been successfully investigated. Such molecules being challenging targets for synthetic chemists, it is indeed of prime importance to guide the experimental efforts towards the most promising targets. In this work, we demonstrated that combining a rigidified azaBODIPY core to smartly selected electro-active groups both near the chromogen and at its periphery may yield a series of dyes absorbing at $c a .900 \mathrm{~nm}$ or above, a feat not yet reached experimentally. It is our hope that the present work will stimulate further research work in that domain.

\section{Acknowledgements}

D.J. is indebted to the Région des Pays de la Loire for financial support in the framework of a recrutement sur poste stratégique. This research used resources of (1) the Interuniversity Scientific Computing Facility located at the University of Namur, Belgium, which is supported by the F.R.S.-FNRS under convention No. 2.4617.07; (2) the GENCI-CINES/IDRIS (Grant c2011085117); (3) the CCIPL (Centre de Calcul Intensif des Pays de Loire). B.L.G. thanks the PSMN (Pôle Scientifique de Modélisation Numérique) at ENS de Lyon for computing facilities. The authors thank Q. Bellier and Dr C. Andraud for fruitful discussions.

\section{References}

1 J. Fabian, H. Nakazumi and M. Matsuoka, Chem. Rev., 1992, 92, $1197-1226$.

2 A. Mishra, R. K. Behera, P. K. Behera, B. K. Mishra and G. B. Behera, Chem. Rev., 2000, 100, 1973-2011.

3 P.-A. Bouit, O. Maury and C. Andraud, Nonlinear Opt., Quantum Opt., 2008, 38, 245-258.

4 K. Kiyose, H. Kojima and T. Nagano, Chem.-Asian J., 2008, 3, 506-515. 
5 A. V. Kulinich and A. A. Ishchenko, Russ. Chem. Rev., 2009, 78, 141-162.

6 G. Qian and Z. Y. Wang, Chem.-Asian J., 2010, 5, 1006-1029.

7 A. Loudet and K. Burgess, Chem. Rev., 2007, 107, 4891-4932.

8 G. Ulrich, R. Ziessel and A. Harriman, Angew. Chem., Int. Ed., 2008, 47, 1184-1201.

9 K. Umezawa, A. Nakamura, H. Makino, D. Citterio and K. Suzuki, J. Am. Chem. Soc., 2008, 130, 1550-1551.

10 A. Burghart, H. J. Kim, M. B. Welch, L. H. Thoresen, J. Reibenspies, K. Burgess, F. Bergstrom and L. B. A. Johansson, J. Org. Chem., 1999, 64, 7813-7819.

11 K. Yamada, T. Toyota, K. Takakura, M. Ishimaru and T. Sugawara, New J. Chem., 2001, 25, 667-669.

12 K. Rurack, M. Kollmannsberger and J. Daub, Angew. Chem., Int. Ed., 2001, 40, 385-387.

13 S. Atilgan, Z. Ekmekci, A. L. Dogan, D. Guc and E. U. Akkaya, Chem. Commun., 2006, 4398-4400.

14 L. A. Burns, A. Vazquez-Mayagoitia, B. G. Sumpter and C. D. Sherrill, J. Chem. Phys., 2011, 134, 084107.

15 M. Wada, S. Ito, H. Uno, T. Murashima, N. Ono, T. Urano and Y. Urano, Tetrahedron Lett., 2001, 42, 6711-6713.

16 Z. Shen, H. Rohr, K. Rurack, H. Uno, M. Spieles, B. Schulz, G. Reck and N. Ono, Chem.-Eur. J., 2004, 10, 4853-4871.

17 S. Goeb and R. Ziessel, Org. Lett., 2007, 9, 737-740.

18 G. Ulrich, S. Goeb, A. De Nicola, P. Retailleau and R. Ziessel, J. Org. Chem., 2011, 76, 4489-4505.

19 J. Chen, A. Burghart, A. Derecskei-Kovacs and K. Burgess, J. Org. Chem., 2000, 65, 2900-2906.

20 S. G. Awuah, J. Polreis, V. Biradar and Y. You, Org. Lett., 2011, 13, 3884-3887.

21 J. Killoran, L. Allen, J. Gallagher, W. Gallagher and D. O'Shea, Chem. Commun., 2002, 1862-1863.

22 A. Gorman, J. Killoran, C. O'Shea, T. Kenna, W. Gallagher and D. F. O'Shea, J. Am. Chem. Soc., 2004, 126, 10619-10631.

23 J. Killoran, S. O. McDonnell, J. F. Gallagher and D. F. O'Shea, New J. Chem., 2008, 32, 483-489.

24 Q. Bellier, S. Pegaz, C. Aronica, B. Le Guennic, C. Andraud and O. Maury, Org. Lett., 2011, 13, 22-25.

25 J. Murtagh, D. O. Frimannsson and D. F. O'Shea, Org. Lett., 2009, 11, 5386-5389.

26 A. Palma, M. Tasior, D. O. Frimannsson, T. T. Vu, R. MéalletRenault and D. F. O'Shea, Org. Lett., 2009, 11, 3638-3641.

27 M. Tasior and D. F. O'Shea, Bioconjugate Chem., 2010, 21, 1130-1133.

28 M. Tasior, J. Murtagh, D. O. Frimannsson, S. O. McDonnell and D. F. O'Shea, Org. Biomol. Chem., 2010, 8, 522-525.

29 P. A. Bouit, K. Kamada, P. Feneyrou, G. Berginc, L. Toupet, O. Maury and C. Andraud, Adv. Mater., 2009, 21, 1151-1154.

30 W. L. Zhao and E. M. Carreira, Angew. Chem., Int. Ed., 2005, 44, $1677-1679$.

31 W. L. Zhao and E. M. Carreira, Chem.-Eur. J., 2006, 12, 7254-7263.

32 A. Loudet, R. Bandichhor, K. Burgess, A. Palma, S. O. McDonnell, M. J. Hall and D. F. O'Shea, Org. Lett., 2008, 10, 4771-4774.

33 R. Gresser, M. Hummert, H. Hartmann, K. Leo and M. Riede, Chem.-Eur. J., 2011, 17, 2939-2947.

34 V. Barone and A. Polimeno, Chem. Soc. Rev., 2007, 36, 1724-1731.

35 E. Runge and E. K. U. Gross, Phys. Rev. Lett., 1984, 52, 997-1000.

36 M. E. Casida, in Time-Dependent Density-Functional Response Theory for Molecules, ed. D. P. Chong, World Scientific, Singapore, 1995, vol. 1, pp. 155-192.

37 M. E. Casida, J. Mol. Struct.: THEOCHEM, 2009, 914, 3-18.

38 M. R. Silva-Junior and W. Thiel, J. Chem. Theory Comput., 2010, 6, 1546-1564.

39 M. Dierksen and S. Grimme, J. Phys. Chem. A, 2004, 108, 10225-10237.

40 D. Jacquemin, E. A. Perpète, O. A. Vydrov, G. E. Scuseria and C. Adamo, J. Chem. Phys., 2007, 127, 094102.

41 D. Jacquemin, E. A. Perpète, G. E. Scuseria, I. Ciofini and C. Adamo, J. Chem. Theory Comput., 2008, 4, 123-135.

42 M. J. G. Peach, P. Benfield, T. Helgaker and D. J. Tozer, J. Chem. Phys., 2008, 128, 044118.

43 M. R. Silva-Junior, M. Schreiber, S. P. A. Sauer and W. Thiel, J. Chem. Phys., 2008, 129, 104103.

44 D. Jacquemin, V. Wathelet, E. A. Perpète and C. Adamo, J. Chem. Theory Comput., 2009, 5, 2420-2435.

45 L. Goerigk, J. Moellmann and S. Grimme, Phys. Chem. Chem. Phys., 2009, 11, 4611-4620.
46 M. Caricato, G. W. Trucks, M. J. Frisch and K. B. Wiberg, J. Chem. Theory Comput., 2010, 6, 370-383.

47 D. Jacquemin, E. A. Perpète, I. Ciofini and C. Adamo, J. Chem. Theory Comput., 2010, 6, 1532-1537.

48 J. Fabian, Dyes Pigm., 2010, 84, 36-53.

49 D. Jacquemin, E. A. Perpète, I. Ciofini, C. Adamo, R. Valero, Y. Zhao and D. G. Truhlar, J. Chem. Theory Comput., 2010, 6, 2071-2085.

50 L. Goerigk and S. Grimme, J. Chem. Phys., 2010, 132, 184103.

51 D. Jacquemin, E. A. Perpète, I. Ciofini and C. Adamo, Theor. Chem. Acc., 2011, 128, 127-136.

52 D. Jacquemin, E. A. Perpète, G. Scalmani, M. J. Frisch, R. Kobayashi and C. Adamo, J. Chem. Phys., 2007, 126, 144105.

53 M. Schreiber, V. Bub and M. P. Fülscher, Phys. Chem. Chem. Phys., 2001, 3, 3906-3912.

54 R. Send, O. Valsson and C. Filippi, J. Chem. Theory Comput., 2011, 7, 444-455.

55 J. Guthmuller, F. Zutterman and B. Champagne, J. Chem. Theory Comput., 2008, 4, 2094-2100.

56 A. E. Masunov, Int. J. Quantum Chem., 2010, 110, 3095-3100.

57 S. Olsen, J. Chem. Theory Comput., 2010, 6, 1089-1103.

58 A. D. Quartarolo, N. Russo and E. Sicilia, Chem.-Eur. J., 2006, 12, 6797-6803.

59 M. J. Frisch, G. W. Trucks, H. B. Schlegel, G. E. Scuseria, M. A. Robb, J. R. Cheeseman, G. Scalmani, V. Barone, B. Mennucci, G. A. Petersson, H. Nakatsuji, M. Caricato, X. Li, H. P. Hratchian, A. F. Izmaylov, J. Bloino, G. Zheng, J. L. Sonnenberg, M. Hada, M. Ehara, K. Toyota, R. Fukuda, J. Hasegawa, M. Ishida, T. Nakajima, Y. Honda, O. Kitao, H. Nakai, T. Vreven, J. A. Montgomery, Jr., J. E. Peralta, F. Ogliaro, M. Bearpark, J. J. Heyd, E. Brothers, K. N. Kudin, V. N. Staroverov, R. Kobayashi, J. Normand, K. Raghavachari, A. Rendell, J. C. Burant, S. S. Iyengar, J. Tomasi, M. Cossi, N. Rega, J. M. Millam, M. Klene, J. E. Knox, J. B. Cross, V. Bakken, C. Adamo, J. Jaramillo, R. Gomperts, R. E. Stratmann, O. Yazyev, A. J. Austin, R. Cammi, C. Pomelli, J. W. Ochterski, R. L. Martin, K. Morokuma, V. G. Zakrzewski, G. A. Voth, P. Salvador, J. J. Dannenberg, S. Dapprich, A. D. Daniels, O. Farkas, J. B. Foresman, J. V. Ortiz, J. Cioslowski and D. J. Fox, Gaussian 09 Revision A.02, Gaussian Inc., Wallingford CT, 2009.

60 D. Jacquemin, E. A. Perpète, I. Ciofini and C. Adamo, Acc. Chem. Res., 2009, 42, 326-334.

61 J. P. Perdew, K. Burke and M. Ernzerhof, Phys. Rev. Lett., 1996, 77, 3865-3868.

62 J. Tao, J. Perdew, V. Staroverov and G. Scuseria, Phys. Rev. Lett., 2003, 91, 146401.

63 V. N. Staroverov, G. E. Scuseria, J. Tao and J. P. Perdew, J. Chem. Phys., 2003, 119, 12129-12137.

64 J. Baker and P. Pulay, J. Chem. Phys., 2002, 117, 1441-1449.

65 A. D. Becke, J. Chem. Phys., 1993, 98, 5648-5652.

66 P. J. Stephens, F. J. Devlin, C. F. Chabalowski and M. J. Frisch, J. Phys. Chem., 1994, 98, 11623-11627.

67 C. Adamo and V. Barone, J. Chem. Phys., 1999, 110, 6158-6170.

68 M. Ernzerhof and G. E. Scuseria, J. Chem. Phys., 1999, 110, 5029-5036.

69 A. D. Boese and J. M. L. Martin, J. Chem. Phys., 2004, 121, 3405-3416.

70 Y. Zhao and D. G. Truhlar, Acc. Chem. Res., 2008, 41, 157-167.

71 T. Tawada, T. Tsuneda, S. Yanagisawa, T. Yanai and K. Hirao, J. Chem. Phys., 2004, 120, 8425-8433.

72 H. Iikura, T. Tsuneda, T. Yanai and K. Hirao, J. Chem. Phys., 2001, 115, 3540-3544.

73 O. A. Vydrov and G. E. Scuseria, J. Chem. Phys., 2006, 125, 234109.

74 O. A. Vydrov, J. Heyd, V. Krukau and G. E. Scuseria, J. Chem. Phys., 2006, 125, 074106.

75 T. Yanai, D. P. Tew and N. C. Handy, Chem. Phys. Lett., 2004, 393, $51-56$.

76 J. D. Chai and M. Head-Gordon, J. Chem. Phys., 2008, 128, 084106.

77 J. D. Chai and M. Head-Gordon, Phys. Chem. Chem. Phys., 2008, 10, 6615-6620.

78 J. Tomasi, B. Mennucci and R. Cammi, Chem. Rev., 2005, 105, 2999-3094.

79 M. Cossi and V. Barone, J. Chem. Phys., 2001, 115, 4708-4717.

80 R. Improta, V. Barone, G. Scalmani and M. J. Frisch, J. Chem. Phys., 2006, 125, 054103.

81 O. Buyukcakir, O. A. Bozdemir, S. Kolemen, S. Erbas and E. U. Akkaya, Org. Lett., 2009, 11, 4644- 4647. 